\title{
ATLAS-ASSISTED TOMOGRAPHY: REGISTRATION OF A DEFORMABLE ATLAS TO COMPENSATE FOR LIMITED-ANGLE CONE-BEAM TRAJECTORY
}

\author{
Ofri Sadowsky ${ }^{1}$, Krishnakumar Ramamurthi ${ }^{2}$, Lotta M. Ellingsen ${ }^{2}$, \\ Gouthami Chintalapani ${ }^{1}$, Jerry L. Prince ${ }^{2}$, Russell H. Taylor ${ }^{1}$ \\ Johns Hopkins University \\ ${ }^{1}$ Department of Computer Science \\ ${ }^{2}$ Department of Electrical and Computer Engineering
}

\begin{abstract}
We present a method to improve the quality of cone-beam tomographic images computed from an intra-operative C-arm scan by adding information from an anatomical atlas. Limited range of $\mathrm{C}$-arm view angles leads to reconstruction artifacts and poor anatomical detail. We propose to complete the missing views with simulated projections of a statistical anatomical model, which is deformably registered to match the data in the $\mathrm{C}$-arm images. This paper presents the methods used to create the atlas and to register it with $\mathrm{x}$-ray images. We compare the results of seven leave-one-out simulated hybrid reconstruction tests on a population of 13 subjects, with "ground-truth" CT, a classical short-scan, and a partial-scan reconstruction.
\end{abstract}

\section{INTRODUCTION}

This paper presents a method for hybrid tomographic reconstruction, which combines data from cone-beam x-ray (Carm) and a statistical anatomical atlas to improve the reconstruction of anatomy and reduce artifacts. C-arm tomography can provide a fast and economical intra-operative 3D imaging. However, in many surgical situations it is impossible to rotate the $\mathrm{C}$-arm gantry over a sufficiently large angular range known as a short scan [1]. Any partial scan that falls short of this requirement yields reconstructions marred with strong artifacts and incorrect anatomical details. Our method compensates for the missing views by combining statistical knowledge of the anatomy into the reconstruction.

The method uses a statistical atlas which contains information about the "average" shape and CT density distribution in the anatomy, and about typical variations in the shape. The atlas is registered to a small set of calibrated subject x-ray images (target images), which may be taken from the partial scan, to recover the 3D pose and shape that best match the targets. Synthetic projections of the recovered model are generated for the missing trajectory angles, and combined with

Email: ofri@cs.jhu.edu

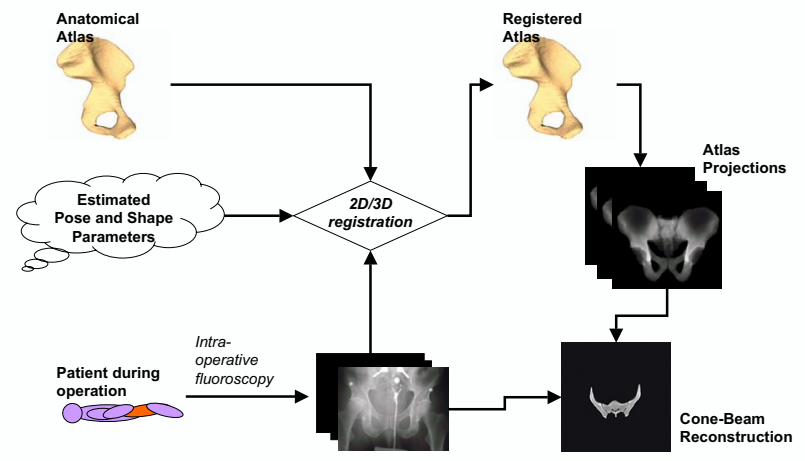

Fig. 1. Schematic flow of the hybrid reconstruction process

the C-arm images as input to a standard cone-beam reconstruction process. The atlas-assisted reconstruction process is illustrated in Fig. 1. We tested the method using a leave-out validation. Results are given in Section 5.

The paper is structured as follows. Section 2 provides background to this paper relating to anatomical modeling and deformable registration. Section 3 explains the atlas creation process. Section 4 describes the registration method we use. Section 5 presents experimental results of reconstruction using a registered atlas. Section 6 discusses the results and concludes.

\section{RELATED WORK}

Although there is an extensive literature on rigid 2D/3D registration of X-ray images to preoperative CT models (e.g., [2, 3, 4]), much less has been published on deformable registration of x-rays to template bone models. The first work we have found is that of Fleute [5], who proposed a deformable 2D/3D method based on a statistical surface model. Subsequently, Yao [6] developed an intensity-based method combining statistical models of shape and CT density. Our method is similar to Yao's in representing bones by tetrahedral meshes, with 
bone shape determined by the vertex coordinates and radiological density within each tetrahedron represented by barycentric Bernstein polynomials. Following Yao, we create a statistical atlas by deforming a standard template mesh to match multiple patient images and performing standard analysis to extract principal components of variation of shape (vertex coordinates) and density (polynomial coefficients), in a manner similar to [7].

\section{STATISTICAL ATLAS CREATION}

This section describes the process of creating the statistical atlas, including the representation of shape and density, and the methods used to construct the model.

The statistical atlas consists of a tetrahedral mesh representing the shape, Bernstein polynomial representing the CT intensities, and displacement matrices that describe deformation modes. We can consider the mesh as given using an array of vertices $V$ and an array of tetrahedral cells $T$. Every vertex is a $3 \mathrm{D}$ vector, and a tetrahedron is defined using four indexes into the vertex array: $\left(i_{0}, i_{1}, i_{2}, i_{3}\right)$. For simplicity, we will usually ignore the indexes and write each tetrahedron as four vertices: $\mathbf{T}_{j}=\left(\mathbf{v}_{0}, \ldots, \mathbf{v}_{3}\right)$. They can be arranged in a matrix of homogeneous coordinates:

$$
M_{\mathbf{T}}=\left[\begin{array}{cccc}
\mathbf{v}_{0} & \mathbf{v}_{1} & \mathbf{v}_{2} & \mathbf{v}_{3} \\
1 & 1 & 1 & 1
\end{array}\right]
$$

The density of each cell is represented as a local Bernstein polynomial function inside the volume of the cell:

$$
f^{j}(\mathbf{u})=\sum_{|\mathbf{k}|=d} \beta_{\mathbf{k}}^{j} B_{\mathbf{k}}^{d}(\mathbf{u})=\sum_{|\mathbf{k}|=d} \beta_{\mathbf{k}}^{j}\left(\begin{array}{l}
d \\
\mathbf{k}
\end{array}\right) u_{0}^{k_{0}} u_{1}^{k_{1}} u_{2}^{k_{2}} u_{3}^{k_{3}}
$$

Here, $\mathbf{u}=\left(u_{0}, u_{1}, u_{2}, u_{3}\right)^{T}=M_{\mathbf{T}}^{-1} \mathbf{x}$ are the barycentric coordinates of a point $\mathbf{x}$ in homogeneous coordinates; $\mathbf{k}=$ $\left(k_{0}, k_{1}, k_{2}, k_{3}\right)$ is the power index of the basis function $B_{\mathbf{k}}^{d}$, with $d$ being the degree of the polynomial; $\left(\begin{array}{l}d \\ \mathbf{k}\end{array}\right)=\frac{d !}{k_{0} ! k_{1} ! k_{2} ! k_{3} !}$ is a multinomial factor; and $\beta$ is a free coefficient.

The atlas creation process can be summarized as follows.

1. Select a "master" CT dataset, $C T_{0}$.

2. Manually label the voxels of the anatomy of interest in $C T_{0}$ (segmentation).

3. Create a "master" tetrahedral mesh, $M_{0}$, that covers the labeled voxels. We use a method developed by Mohamed [8]. The spatial resolution of the mesh is defined by the user, and typically every cell contains many $\mathrm{CT}$ voxels. The mesh has $n_{v}$ vertices and $n_{t}$ tetrahedra.

4. For each instance $C T_{i}$ in "subject" datasets, find an elastic transformation $D_{i}$ that maps the voxels of $C T_{0}$ to corresponding voxels in $C T_{i}$. We use the registration method in [9].
5. Apply the deformation $D_{i}$ to the vertices of $M_{0}$ to obtain a "subject" mesh $M_{i}$. Note that all the meshes created this way have identical graphs.

6. Take all the mesh instances as shape vectors of size $3 n_{v}$, including $M_{0}$, and perform principal component analysis (cf. [10]) to obtain a mean shape $\bar{M}$ and variation modes $\left\{Y_{i}\right\}$ of the shape distribution. The variation modes are applied to the mean shape with different weights to create shape instances. Note that the vertices of the final mesh $\bar{M}$ are given in some arbitrary coordinate system $F_{\text {model }}$. The registration process (Section 4) maps it to the space of subject images.

7. In each mesh instance $M_{i}$, for each tetrahedron $\mathbf{T}_{j}$, find polynomial coefficients $\left\{\beta_{\mathbf{k}}^{j}\right\}$ to approximate the density values in $C T_{i}$ in the area occupied by $\mathbf{T}_{j}$. Store all the coefficients as a matrix $\Gamma_{i}$.

8. In the current implementation of the atlas, we use the mean of the density functions $\left\{\Gamma_{i}\right\}$ as the atlas density.

\section{REGISTRATION}

The deformable registration process matches the atlas to a set of calibrated patient projections to recover approximated pose and shape of the patient's anatomy. A calibrated image is given as the tuple $(I, F, P)$, with $I$ being the actual image (pixel values), $F$ as a rigid transformation representing the camera pose (extrinsic parameters), and $P$ as parameters of a projection transformation from the 3D space to image pixels (intrinsic parameters). Both $F$ and $P$ are required for a correct tomographic reconstruction, and we assume that they are given for each X-ray image, with respect to some constant reference frame $F_{\text {world }}$, e.g., the room.

The registration involves finding a rigid transformation $F_{\text {reg }}$ that relates the model coordinate system $F_{\text {model }}$ to the reference frame:

$$
F_{\text {world }}=F_{\text {reg }} \cdot F_{\text {model }}
$$

It also involves finding a set of weights $\Lambda=\left\{\lambda_{i}\right\}$ that are applied to the deformation modes to create a patient-specific instance of the atlas:

$$
S_{\Lambda}=\bar{M}+\sum_{i=1}^{n_{\text {modes }}} \lambda_{i} Y_{i}
$$

Our goal is to approximate the shape of the patient's anatomy by finding the right combination of $\lambda_{i}$ 's, and the pose of the anatomy. We do this by optimizing a mutual information $[11,6]$ similarity measure between the patient images and computed projections (DRR's) of the model.

$$
\left(F_{\text {reg }}, \Lambda_{\text {reg }}\right)=\arg \max _{(F, \Lambda)} \sum_{j} M I\left(I_{j}, \operatorname{DRR}\left(F \cdot S_{\Lambda}\right)\right)
$$




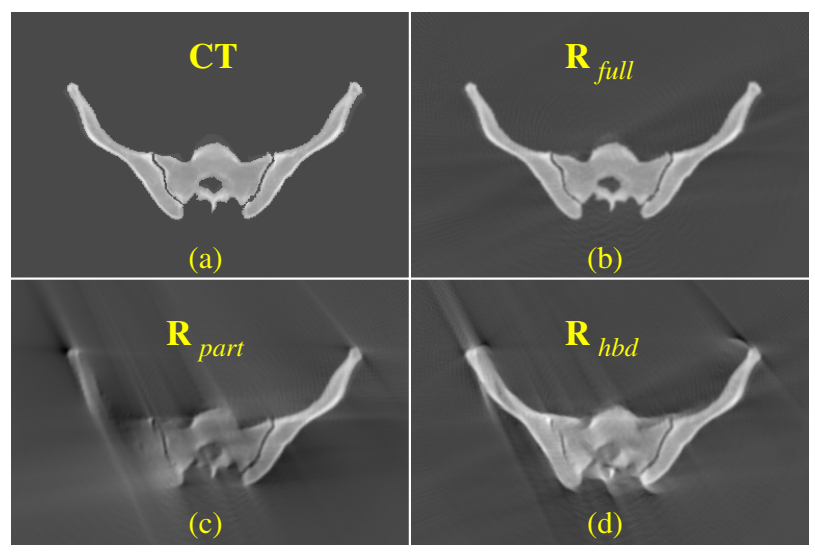

Fig. 2. Visual comparison of different reconstruction methods. (a) A slice from a CT scan. (b) A slice reconstructed from a $207^{\circ}$ short-scan. (c) A slice reconstructed from a $120^{\circ}$ partial scan. (d) A slice from a $120^{\circ}$ partial-scan and $87^{\circ}$ registered atlas projections. All dynamic ranges have been scaled uniformly so that zero maps to the gray background in (a), and white is the maximal intensity value.

We have recently developed a fast method for generating DRR's of a deformable tetrahedral mesh [12], which facilitates this registration within a reasonable time frame. The optimal values are searched for using the Downhill Simplex algorithm, implemented in the VNL package [13].

\section{EXPERIMENTS AND RESULTS}

Our validation experiments consist of leave-one-out tests. Given $N$ subject CT datasets, we pick $N-1$ and create a statistical atlas, which is registered with images of the $N^{t h}$ subject. We generate DRR projections of the target dataset along a trajectory of $k$ degrees ( $k=180^{\circ}+$ cone angle, i.e., a short scan), in increments of $1^{\circ}$, and reconstruct the volume in three ways:

$\mathbf{R}_{\text {full }}$ Using all $k$ projections from the CT (short scan).

$\mathbf{R}_{\text {part }}$ Using a partial scan of $l$ degrees of CT projections.

$\mathbf{R}_{h b d}$ Using a partial scan of $l$ degrees of CT projections, and completing them with $k-l$ projections of the registered atlas (hybrid reconstruction).

For the data shown here, an atlas of the pelvis bones was created, with $N=13$. The registration was done on images of size $256 \times 256$ pixels, and the reconstruction used a size of $512 \times 512$. The registration used three images, at angles $0^{\circ}$, $90^{\circ}$ and $120^{\circ}$ in the $X Y$ plane. In our cone-beam simulations, the focal-length was set to $750 \mathrm{~mm}$ and the cone-angle $=27^{\circ}$ $\left(k=207^{\circ}\right)$.

The reconstruction from $2 \mathrm{D}$ projections was done using a filtered backprojection technique that was used in [14]. However in this case, we compensate only for missing views, and

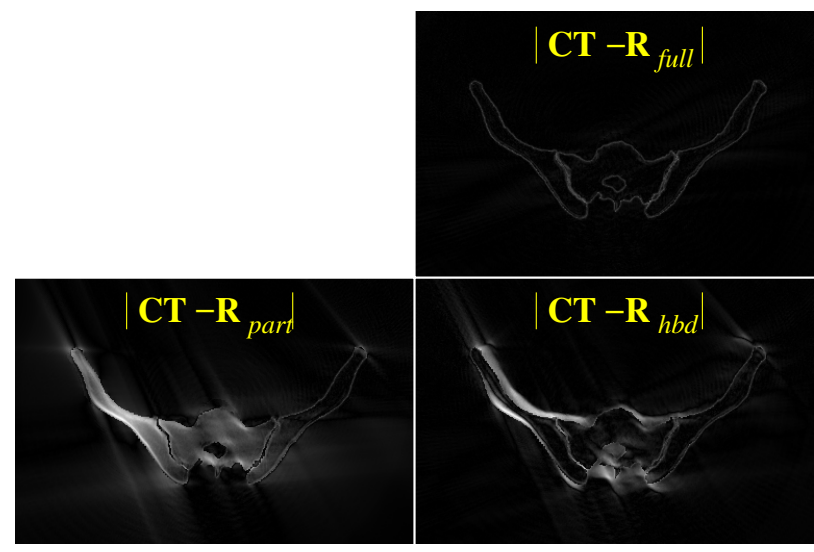

Fig. 3. Scaled absolute differences between the cone-beam reconstructed images and the CT image in Fig. 2. Zero is mapped to black, and the largest absolute difference is white. Note that the scale of brightness is not as Fig. 2. Comparing the partial-scan and hybrid reconstruction to the short scan results in very similar absolute difference images.

the images are not truncated. Note that our atlas covers bone only, and correspondingly the DRRs in the registration and reconstruction were computed from a segmented CT dataset.

Examples of reconstructed cross-sections are shown in Fig. 2. The figure shows corresponding tomographic slices of the mid-iliac region from a segmented CT image (a), a cone-beam reconstruction from a short scan (b), a reconstruction from partial scan (c), and a hybrid reconstruction (d). The gray levels were scaled uniformly. Reconstructed images usually contain some negative artifacts, hence the gray background in image (a), which represents the CT number zero. For further understanding of these results, Figure 3 shows the absolute differences between the cone-beam reconstructions and the CT dataset as intensity levels.

For a quantitative analysis of the results, we compute the root-mean-square (RMS) difference $E$ between the volumes as follows:

$$
E\left(I_{1}, I_{2}\right)=\sqrt{\frac{1}{n_{v o x}} \sum_{v \in V}\left(I_{1}(v)-I_{2}(v)\right)^{2}}
$$

Here, $n_{v o x}$ is the number of voxels in both volumetric images $I_{1}$ and $I_{2} . V$ is the domain of voxels. Lower RMS values mean greater similarity between the compared images. Note that positive and negative background reconstruction artifacts are included in the RMS difference.

We performed seven leave-one-out tests on our 13-subject population. The RMS results are summarized in Table 1, including the following elements: CT: the segmented target CT dataset; $\mathbf{R}_{\text {full }}$ : a short-scan reconstruction; $\mathbf{R}_{\text {part }}$ : a partialscan reconstruction; $\mathbf{R}_{h b d}$ : a hybrid reconstruction. The Table compares all three methods against the "ground truth" $\mathrm{CT}$, and the partial-scan and hybrid methods against the short 


\begin{tabular}{|c|c|c|c|c|c|c|}
\hline \# & $\begin{array}{l}\text { (1) } \\
\text { CT } \\
\mathbf{R}_{\text {full }}\end{array}$ & $\begin{array}{l}\text { (2) } \\
\mathbf{C T} \\
\mathbf{R}_{\text {part }}\end{array}$ & $\begin{array}{l}\text { (3) } \\
\text { CT } \\
\mathbf{R}_{h b d}\end{array}$ & $\begin{array}{l}\text { (4) } \\
\mathbf{R}_{\text {full }} \\
\mathbf{R}_{\text {part }}\end{array}$ & $\begin{array}{l}\text { (5) } \\
\mathbf{R}_{\text {full }} \\
\mathbf{R}_{\text {hbd }}\end{array}$ & $\begin{array}{l}\text { (4)-(5) } \\
/ \quad{ }^{2} \\
\%\end{array}$ \\
\hline 1 & 39.0 & 108.9 & 73.9 & 102.3 & 63.1 & 38.3 \\
\hline 2 & 37.4 & 115.5 & 105.7 & 109.1 & 98.0 & 10.2 \\
\hline 3 & 39.1 & 118.5 & 68.6 & 111.6 & 56.6 & 49.3 \\
\hline 4 & 33.4 & 98.0 & 70.3 & 91.3 & 61.4 & 32.7 \\
\hline 5 & 38.1 & 108.0 & 79.3 & 101.2 & 71.1 & 29.7 \\
\hline 6 & 28.0 & 104.0 & 82.4 & 97.5 & 74.3 & 23.8 \\
\hline 7 & 44.0 & 130.3 & 94.9 & 122.7 & 83.4 & 32.0 \\
\hline$\mu$ & 37.0 & 111.9 & 82.2 & 105.1 & 72.6 & 31.0 \\
\hline
\end{tabular}

Table 1. Root-mean-square (RMS) difference measure between different reconstruction methods. The differences are expressed in Hounsfeld units. The last column shows the difference between column 5 and column 4 as a percentage of column 4 . The last row shows the mean of RMS differences, and its respective relative difference.

scan. All the RMS numbers are given in Hounsfeld units. The last column contains the percent of change in RMS between columns 5 and 4, with respect to column 4 . The reconstruction included 200 slices: 100 above and 100 below the plane of rotation. The voxel size is $0.9375 \mathrm{~mm}$ in each direction, and the slice size is $512 \times 512$ pixels.

\section{DISCUSSION AND CONCLUSION}

We have shown an average of $31 \%$ reduction in RMS difference going from a partial scan to atlas-assisted tomography, taken with respect to the "ideal" short-scan reconstruction, and a significant visual improvement in reconstructing anatomical detail. In the worst case the RMS difference was $10.2 \%$, and in the best case $49.3 \%$. We attribute this variability to varying qualities of the registration results, and expect the results to be more regular when a larger population is included, and when density variations are added to the atlas. We are in a continuing our work towards these improvements.

The quality of the atlas deformable registration, which is highly dependent on the fidelity of anatomical representation by the atlas, is a key factor in the reconstruction. It is expected to be further improved by finding the optimal number of views the optimal view directions. Other experiments must be performed, such as registration with truncated fieldof-view images and registration with real x-ray images. These are subjects of our continuing research. Nevertheless, we consider the results presented here, which were obtained with a small-population atlas, a very good starting point.

\section{ACKNOWLEDGMENTS}

We would like to thank the following people for their assistance in this work: Dr. Ashraf Mohamed, Pauline Pelletier, Dinggang Shen, Na Song, Yiqiang Zhan. The pelvis datasets were given to us by Dr's. Ted DeWeese and Lee Myers. This work was supported in part by NSF ERC Grant EEC9731478, by NIH/NIBIB research grant R21-EB003616, and by Siemens Corporate Research.

\section{REFERENCES}

[1] Dennis L. Parker, "Optimal short scan convolution reconstruction for fanbeam CT," Physics in Medicine and Biology, vol. 9, pp. 254-257, 1982.

[2] A Hamadeh, P Sautot, S Lavallee, and P Cinquin, "Towards automatic registration between ct and $\mathrm{X}$-ray images: cooperation between $3 \mathrm{~d} / 2 \mathrm{~d}$ registration and $2 \mathrm{~d}$ edge detection," in Second Annual International Symposium on Medical Robotics and Computer Assisted Surgery, Baltimore, MD USA, 1995, pp. 39-46.

[3] L Zollei, E Grimson, A Norbash, and W Wells, "2d-3d registration of x-ray fluoroscopy and ct images," in IEEE CVPR, 2001.

[4] E v. d. Kraats, G Penney, D Tomazevic, T v. Walsum, and W Niessen, "Standardized evaluation of $2 \mathrm{~d}-3 \mathrm{~d}$ registration," in MICCAI 2004, St. Malo, France, 2004, pp. 574-581, Springer Lecture Notes in Computer Science.

[5] M Fleute and S Lavallee, "Nonrigid 3-d/2-d registration of images using statistical models," in MICCAI 99, Cambridge, UK, 1999, pp. 138-147, Springer Lecture Notes in Computer Science.

[6] Jianhua Yao, A statistical bone density atlas and deformable medical image registration, Ph.D. thesis, Johns Hopkins University, 2002.

[7] T F Cootes, C Beeston, G J Edwards, and C J Taylor, "A unified framework for atlas matching using active appearance models," in IPMI 1999. 1999, pp. 323-333, Springer.

[8] Ashraf Mohamed and Christos Davatzikos, "An approach to $3 \mathrm{~d}$ finite element mesh generation from segmented medical images," in IEEE International Symposium on Biomedical Imaging (ISBI), 2004.

[9] L.M. Ellingsen and J.L. Prince, "Mjolnir: Deformable image registration using feature diffusion," in Proc. SPIE Medical Imaging, 2006, accepted for publication.

[10] T F Cootes, C J Taylor, D H Cooper, and J Graham, "Active shape models - their training and application," Computer Vision and Image Understanding, vol. 6, no. 1, pp. 38-59, 1995.

[11] P. Viola and W.M. Wells, "Alignment by maximization of mutual information," International Journal of Computer Vision, vol. 24, pp. 137-154, 1997.

[12] Ofri Sadowsky, Jonathan D. Cohen, and Russell H. Taylor, "Rendering tetrahedral meshes with higher-order attenuation functions for digital radiograph reconstruction," in Proceedings of IEEE Visualization 2005.

[13] “The vxl homepage,” On the Web: http://vxl.sourceforge.net .

[14] K. Ramamurthi and J. Prince, "Tomographic reconstruction for truncated cone beam data using prior CT information," in Sixth International Conference: Medical Imaging Computing and Computer-Assisted Intervention (MICCAI), 2003, pp. 134-141. 